\title{
PANCs - PLANTAS ALIMENTÍCIAS NÃO CONVENCIONAIS E SEUS BENEFÍCIOS NUTRICIONAIS
}

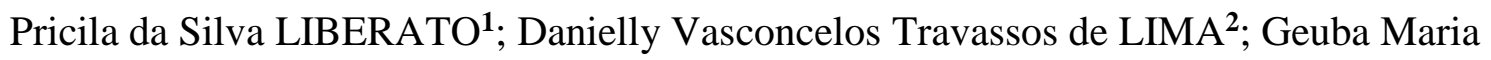
Bernardo da SILVA ${ }^{3}$

\author{
${ }^{1}$ Faculdade Internacional da Paraíba (FPB), Laureate International Universities, João Pessoa, Brasil. E-mail: \\ prisliberato@hotmail.com \\ ${ }^{2}$ Professora/Faculdade Internacional da Paraíba (FPB), Laureate International Universities, João Pessoa, Brasil. E- \\ mail: daniellytravassos@gmail.com \\ ${ }^{3}$ Universidade Estadual da Paraíba (UEPB), Brasil. E-mail: geubasilva@gmail.com
}

Resumo. O conceito de alimentação muda conforme o conhecimento da população sobre alimentos saudáveis e seus benefícios. A busca por uma vida saudável leva as pessoas a buscarem novas formas de alimentação tendo em vista sua funcionalidade e sustentabilidade. O presente trabalho teve como objetivo fazer uma revisão bibliográfica acerca do tema PANC, identificar as propriedades funcionais, nutrientes, valor nutricional, formas de consumo e inserção na alimentação humana. Realizou-se uma busca sistemática da bibliografia publicada nas bases de dados na "Literatura Latino-Americana e do Caribe em Ciências da Saúde" (LILACS), "Medical Literature Analysis and Retrieval System On-line" (MEDLINE) e "Google Acadêmico". Os descritores utilizados para a busca foram: Plantas; Nutricional; Funcional; Antioxidantes; Anti-inflamatório; Consumo; Benefícios. Foram selecionados um total de 20 artigos publicados entre os anos de 2009 a 2019, nos idiomas português, inglês e espanhol. Nesse trabalho identificou-se que de um modo geral as PANCs em sua maioria apresentam fator nutricional elevado, aumentando para o indivíduo que as consomem a ingestão diária vitaminas e minerais essenciais ao desenvolvimento humano, como visto em alguns casos são capazes de substituir as hortaliças convencionais, podendo ser inserida na alimentação diária, cultivadas em pequenos espaços e jardins, podendo também ser comercializada gerando uma fonte de renda. Conclui-se que se faz necessário que os pesquisadores aprofundem seus estudos em relação ao tema, pois existe uma grande diversidade a ser explorado. Por fim, verificou-se a necessidade da população adquiri mais conhecimento para que essas PANCs sejam inseridas na sua alimentação diária.

Palavras-chave: Plantas; Nutrição Funcional; Antioxidantes; Anti-inflamatório; Consumo; Benefícios.

\section{UFPs - UNCONVENTIONAL FOOD PLANTS AND THEIR NUTRITIONAL BENEFITS}

Abstract. The concept of food changes according to the population's knowledge of healthy foods and their benefits. The quest for a healthy life leads people to seek new ways of eating in view of its 
functionality and sustainability. The present work had as objective to make a bibliographic review about the PANC theme, to identify the functional properties, nutrients, nutricional value, forms of consumption and insertion in human food. A systematic search of the bibliography published in the "Literatura Latino-Americana e do Caribe em Ciências da Saúde" (LILACS), "Medical Literature Analysis and Retrieval System On-line (MEDLINE)", and "Google Scholar" was carried out. The descritora used for the search were: Plants; Nutritional; Functional; Antioxidants; Anti-inflammatory; Consumption; Benefits. A total of 20 articles were published between the years 2009 and 2019, in Portuguese, English and Spanish. In this work, it was identified that in general the UFP in most of them present a high nutritional factor, increasing for the individual that consumes the daily intake vitamins and minerals essential to human development, as seen in some cases are able to substitute the conventional vegetables, and can be inserted in daily food, grown in small spaces and gardens, and can also be commercialized generating a source of income. It is concluded that it is necessary that the researchers deepen their studies in relation to the subject, because there is a great diversity to be explored. Finally, it was verified the need of the population acquired more knowledge so that these UFPs are inserted in their daily food.

Keywords: Plants; Functional Nutrition; Antioxidants; Anti-inflammatory; Consumption; Benefits.

\section{PANCS - PLANTAS DE ALIMENTOS NO CONVENCIONALES Y SUS BENEFICIOS NUTRICIONALES}

Resumen. El concepto de alimentos cambia según el conocimiento de la población sobre alimentos saludables y sus beneficios. La búsqueda de una vida saludable lleva a las personas a buscar nuevas formas de comer, en vista de su funcionalidad y sostenibilidad. El presente trabajo tuvo como objetivo realizar una revisión bibliográfica sobre el tema PANC, identificar las propiedades funcionales, nutrientes, valor nutricional, formas de consumo e inserción en alimentos humanos. Se llevó a cabo una búsqueda sistemática de la bibliografía publicada en la "Literatura Latino-Americana e do Caribe em Ciências da Saúde" (LILACS), "Medical Literature Analysis and Retrieval System On-line" (MEDLINE) y "Google Académico". Los descriptores utilizados para la búsqueda fueron: Plantas; Nutricional; Funcional; Antioxidantes; Antiinflamatorio; Consumo; Beneficios Se publicaron un total de 20 artículos entre los años 2009 y 2019, en portugués, inglés y español. En este trabajo se identificó que, en general, los PANCs en su mayoría presentan un alto factor nutricional, aumentando para el individuo que los consume la ingesta diaria de vitaminas y minerales esenciales para el desarrollo humano, como se puede observar en algunos casos para reemplazar las verduras convencionales. , y puede insertarse en alimentos diarios, cultivarse en espacios pequeños y jardines, y también puede comercializarse generando una fuente de ingresos. Se concluye que es necesario que los investigadores profundicen sus estudios en relación con el tema, ya que hay una gran diversidad por explorar. 
Finalmente, se verificó la necesidad de que la población adquiriera más conocimientos para que estos PANCs se insertaran en su alimentación diaria.

Palabras clave: Plantas; Nutrición funcional; Antioxidantes; Antiinflamatorio; Consumo; Beneficios.

\section{INTRODUÇÃO}

O conceito de alimentação muda conforme o conhecimento da população sobre a alimentos saudáveis e seus benefícios. A busca por uma vida saudável leva as pessoas a buscarem novas formas de alimentação tendo em vista sua funcionalidade e sustentabilidade, nesse sentido, podemos perceber em vários setores mudanças profundas, que vieram crescendo nas últimas décadas e levam ao indivíduo o retorno a vida natural (COSTA, 2012).

Uma das alternativas por uma alimentação mais equilibrada e saudável é o consumo de alimentos não industrializados, ou seja, uma alimentação composta por proteínas, gorduras, carboidratos, fibras, vitaminas e minerais entre outros, os quais podem ser encontrados nos cerais, frutas, legumes, carnes e verduras.

Diante desse contexto, as Plantas Alimentícias Não Convencionas (PANCs), apresentam-se como uma ótima fonte nutricional e funcional para a alimentação humana. $\mathrm{O}$ termo PANC foi criado em 2008 pelo Biólogo e Professor Valdely Ferreira Kinupp e refere-se a todas as plantas que possuem uma ou mais partes comestíveis, sendo elas espontâneas ou cultivadas, nativas ou exóticas que não estão incluídas em nosso cardápio cotidiano (KELEN, et al., 2015).

São caracterizadas como PANCs as plantas que ainda não foram completamente estudadas por parte da comunidade técnico-científica e/ou exploradas pela sociedade como um todo, resultando em consumo regional e apresentando dificuldade de aceitação e consumo para as demais regiões do país (BRASIL, 2010).

No Brasil existem pelo menos 3 mil espécies conhecidas de PANCs, estudos indicam que cerca de $10 \%$ da flora sejam de plantas alimentícias (KELEN et al., 2015).

Conforme dados da Organização das Nações Unidas para Alimentação e Agricultura (FAO), calcula-se que em todo o planeta o número de plantas consumidas pelo homem caiu de 10 mil para 170 nos últimos cem anos (LIRA, 2018).

Segundo Pedrosa et al. (2012), são plantas que crescem espontaneamente e são típicas em determinadas regiões, geralmente são cultivadas por agricultores familiares, principalmente entre os povos mais tradicionais, pois seu manejo e cultivo são repassados de geração para geração, e na maioria das vezes o consumo ocorre na própria família, sem intuito comercial. Como também são utilizadas na Fitoterapia e medicina popular, as PANCs exercem o papel de 
alimentos funcionais, pois apresentam na sua composição, vitaminas essenciais, fibras, antioxidantes e sais minerais, ou seja, todos os nutrientes necessários para o nosso organismo (KELEN, et al. 2015).

As Plantas Alimentícias não Convencionais, são plantas que poderiam fazer parte do nosso consumo diário. Porém, devido a falta de conhecimento por grande parte da população, muitas dessas plantas são caracterizadas como ervas daninhas, podendo ser facilmente encontradas na natureza, consideradas como mato e ignoradas.

Por possuírem um alto valor nutritivo, sendo fontes de sais minerais, vitaminas, carboidratos e proteínas, por que não as introduzir na alimentação diária?

Tais questionamentos nos direcionaram ao desenvolvimento dessa pesquisa tem por objetivo fazer uma revisão bibliográfica acerca do tema PANC, identificar as propriedades funcionais, nutrientes, valor nutricional, formas de consumo e inserção na alimentação humana.

\section{MATERIAIS E MÉTODOS}

Realizou-se uma busca sistemática da bibliografia publicada nas bases de dados na "Literatura Latino-Americana e do Caribe em Ciências da Saúde" (LILACS), "Medical Literature Analysis and Retrieval System On-line” (MEDLINE) e "Google Acadêmico". Os descritores utilizadas para a busca foram: Plantas; Nutricional; Funcional; Antioxidantes; Anti-inflamatório; Consumo; Benefícios.

Foram selecionados artigos científicos publicados entre os anos de 2009 a 2019, nos idiomas português, inglês e espanhol. Quanto aos critérios de exclusão, excluíram-se artigos científicos não relevantes que abordavam outras formas de plantas e hortaliças que não faziam parte das PANCs e artigos publicados fora dos anos limites.

Quanto ao processo de seleção, somadas as bases escolhidas e os critérios propostos obteve-se o número de 20 artigos pertinentes ao tema e ao período proposto.

A seleção dos artigos seguiu as seguintes etapas: identificação de trabalhos repetidos, leitura dos descritores, leitura dos títulos, leitura dos resumos, análise metodológica e exclusão de publicações de menos correlação com o objetivo da pesquisa num total de 5 artigos.

\section{RESULTADOS E DISCUSSÕES}

A grande maioria das PANCs não são cultivadas, crescem espontaneamente sem que sejam plantadas, podem ser cultivadas, não precisam de muita atenção apenas cuidados básicos em relação aos demais cultivos. As PANCs são plantas independentes pois possuem maior adaptabilidade devido sua variação genética. As hortaliças e legumes também fazem parte das 
PANCs, pois o cultivo que é comum em uma região pode não ser em outra, isso quer dizer que o termo PANC pode ser utilizado para o que não convencional em uma região. Inserir as plantas na alimentação significa variar o cardápio, aprender novos sabores aliados a uma alimentação rica em diversos nutrientes necessários para a homeostase do nosso organismo, as PANC são uma alternativa para uma alimentação adequada, saudável e consciente (KINUPP, 2014).

De acordo com Fischer e Garnett (2016),

Atualmente o aumento do consumo de produtos processados tem afastado a população de uma alimentação saudável levando a um consumo demasiado aliado ao desperdício. Os sistemas alimentares atuais estão sendo cada vez mais desafiados a fornecer alimentos adequados, seguros, diversificados e ricos em nutrientes necessários a uma alimentação saudável.

Tidas como excelentes fontes de nutrientes, vitaminas e sais minerais, as PANCs também possuem características que conferem propriedades antioxidantes, anti-inflamatórias e ação terapêutica, o consumo de tais plantas e hortaliças deve ser realizado respeitando suas características e formas de preparo para que tais efeitos sejam obtidos com segurança. Deve-se, também, aprofundar os conhecimentos e conduzir mais estudos acerca da possível presença de fotoquímicos tóxicos ou fatores antinutricionais que algumas PANCs podem apresentar se consumidas de forma inapropriada (PASCHOAL; SOUZA, 2015).

Dentro da perspectiva de revisão bibliográfica sobre as PANCs, seus benefícios, valor nutricional e cuidados de utilização, com embasamento em artigos científicos e literaturas especializadas no assunto trazem os/as várias faces das PANCs.

A Embrapa em 2018 pulicou um artigo relacionando as PANC seus benefícios e formas de utilização. Foram citadas plantas como Açafrão-da-terra (Cúrcuma longa) que é rica em minerais, vitamina $\mathrm{C}$ e A, ácido fólico, riboflavina e é utilizada na produção de lacticínios e como condimento, a Araruta (Maranta arundinacea) bastante utilizada por pessoas que possuem restrição alimentar relacionada à doença celíaca, pois seria um alimento de fácil digestão. Também citado neste artigo o Hibisco (Hibiscus sabdariffa) bastante utilizado no Norte do Brasil, sendo mais comumente aproveitado folhas e no resto do mundo onde também é bastante apreciada e consumido. O inhame comumente utilizado no Nordeste foi citado como PANC, é um alimento muito ricos em vários nutrientes como fosforo, cálcio, ferro e vitaminas do complexo B, é um carboidrato excelente pois possui baixo índice glicêmico. 
Conforme relata Maria Filho (2016), em seu artigo sobre as PANC, aborda algumas plantas de diversas partes do país como a Taioba (Xanthosoma sagittifolium taioba E.G. Gonç) encontrada Minas Gerais e Rio de Janeiro, as folhas possuem alto teor de fibras, carotenoides, vitamina $\mathrm{C}$, ferro e cálcio, bem como potássio, fósforo e cobre, devido a presença dos alcaloides se consumidas sem ferver pode ser hepatotóxicos, nefrotóxicos, imunotóxicos e neurotóxicos. A Mangaba (Hancornia speciosa Gomes) relacionada nesse artigo como PANC está presente nas regiões Norte, Nordeste e Centro-Oeste, é rica em vitaminas C e A, folato, betacaroteno, fenóis, flavonoides e fibras, devido sua composição tem efeito anti-hipertensivo.

Outra planta bastante conhecida é a Ora-pro-nóbis (Pereskia aculeata Mill.), encontrada na região Sudeste, contém em sua constituição cálcio, magnésio, vitamina A, vitamina B9, vitamina $\mathrm{C}$, triptofano, zinco, fibra, possui um grande potencial proteico. Essa PANC contém oxalato em sua composição, devem ser preparadas em alta temperatura para inibir o ativo.

A Universidade Federal do Rio Grande do Sul no ano de 2015 publicou uma cartilha acerca das PANCs com o objetivo de promover uma maior consciência ambiental sobre a necessidade de cuidados com a agrobiodiversidade e alimentação saudável e sustentável, atingindo a soberania alimentar (KELEN et al., 2015).

Foram retratadas as PANCs de várias partes do Brasil, como a Bertalha (Anredera cordifolia), cresce no Sul do Brasil é fonte de ferro e vitaminas A, B e C. Apresentar fator anti microbiano, Capuchinha (Tropaeolum majus), floresce no México e Peru, é fonte em vitamina $\mathrm{C}$, antocianina, carotenoides e flavonoides, possui propriedade expectorante, anti- oxidante, anti - inflamatório e hipotensor, é tido como calmante natural. Podemos ainda citar uma PANC bastante conhecida, a Dente-de-leão (Taraxacum officinale) natural da Ásia e Europa, cresce espontaneamente em jardins e hortas, é fonte de vitaminas A, B e C, ferro e potássio, são diuréticas. A seguir relacionamos algumas PANCs as quais podemos identificar o nome cientifico, o nome popular como são conhecidas, seus nutrientes e a formas de utilização (Tab. 1).

Tabela 1. Plantas Alimentícias não Convencionais PANCs.

\begin{tabular}{llllll}
\hline $\begin{array}{l}\text { Nome } \\
\text { cientifico }\end{array}$ & Nome & Nutrientes & Benefícios & Forma de utilização \\
\hline Limnocharis & Couve & Cálcio e Proteína & Pode ser usada na As folhas jovens, os botões \\
flava & d’água & & culinária, substitui & o florais, as flores e os pendiculos \\
& & couve comum, o são comestíveis podem ser & espinafre e o brócolis & cozidos, refogados e fritos.
\end{tabular}




\begin{tabular}{|c|c|c|c|c|}
\hline $\begin{array}{l}\text { Alternanthera } \\
\text { philoxeroides }\end{array}$ & $\begin{array}{l}\text { Bredo-d } \\
\text { ‘água }\end{array}$ & $\begin{array}{l}\text { Proteína vegetal } \\
\text { negligenciada, } \\
\text { magnésio, } \\
\text { manganês, zinco, } \\
\text { enxofre e boro. }\end{array}$ & $\begin{array}{l}\text { Serve de forrageira para } \\
\text { o gado e outros animais, } \\
\text { as folhas podem ser } \\
\text { usadas no preparo de } \\
\text { ração, pode ser } \\
\text { consumida } \\
\text { verdura. }\end{array}$ & $\begin{array}{l}\text { Culinária } \\
\text { Pode ser transformada em bolos, } \\
\text { pães, suflês etc. }\end{array}$ \\
\hline $\begin{array}{l}\text { Plumeria } \\
\text { rubra }\end{array}$ & $\begin{array}{l}\text { Jasmim- } \\
\text { manga }\end{array}$ & $\begin{array}{l}\text { As flores são ricas } \\
\text { em antocianinas, } \\
\text { possui } \quad \text { ação } \\
\text { antioxidante. Uso } \\
\text { medicinal, rica em } \\
\text { alcaloides } \\
\text { glicosídeos. }\end{array}$ & $\begin{array}{l}\text { As folhas secas podem } \\
\text { ser usadas como chá. As } \\
\text { flores são usadas na } \\
\text { indústria de cosmético na } \\
\text { fabricação de hidratantes } \\
\text { e cremes corporais. } \\
\text { A espécie do gênero } \\
\text { plumeria tem alcaloides } \\
\text { tóxicos. }\end{array}$ & $\begin{array}{l}\text { As flores podem ser consumidas } \\
\text { como doces, geleias, temperos. }\end{array}$ \\
\hline $\begin{array}{l}\text { Dahlia } \\
\text { pinnata }\end{array}$ & $\begin{array}{l}\text { Dália- } \\
\text { de- } \\
\text { jardim }\end{array}$ & $\begin{array}{l}\text { Possui inulina, } \\
\text { oligrofutosacarideo, } \\
\text { uso medicinais e } \\
\text { dietéticos }\end{array}$ & $\begin{array}{l}\text { Pode ser consumida } \\
\text { como hortaliça. Usada na } \\
\text { fabricação de xarope e } \\
\text { usada como forrageira } \\
\text { para animais. }\end{array}$ & $\begin{array}{l}\text { Da raiz é produzido um extrato } \\
\text { que pose ser utilizado como } \\
\text { bebida aromatizante, pode ser } \\
\text { diluído em agua ou leite, ou } \\
\text { como cobertura de sorvetes. }\end{array}$ \\
\hline $\begin{array}{l}\text { Coronopus } \\
\text { didymus }\end{array}$ & Mentruz & $\begin{array}{l}\text { Umidade, fibras } \\
\text { proteínas, lipídios, } \\
\text { carboidratos, } \\
\text { cinzas, energia, } \\
\text { vitamina }\end{array}$ & $\begin{array}{l}\text { Pode ser usada como } \\
\text { hortaliça. }\end{array}$ & $\begin{array}{l}\text { Servida como salada, remédios } \\
\text { caseiros. As folhas podem ser } \\
\text { consumidas cruas ou cozidas. }\end{array}$ \\
\hline
\end{tabular}

Fonte: Os autores.

Descrevemos, portanto, uma amostra de cinco PANCs que estão presentes no nosso dia a dia, porém, são consideradas como "matos", outras utilizadas apenas como decorativas e outras usadas apenas como remédios caseiros. Nesse sentido, identificamos que a população necessita de mais conhecimento em relação aos benefícios que essas pancs podem trazer, tanto a nível de consumo, como a comercialização.

Dentro desse contexto, um grande referencial de PANC foi publicado em 2014, "Plantas Alimentícias Não Convencionais (PANC) no Brasil”, esse guia identifica 351 espécies de plantas, informa os aspectos nutricionais e apresenta receitas e formas de consumir esse 
alimento. Podemos destacar a Siriguela (Spondias purpúrea L.P) uma iguaria presente no Norte e Nordeste do Brasil, a seriguela na verdade se trata de um fruto bastante utilizado em sucos, polpas. As folhas possuem alto teor de proteína e podem ser consumidas cruas ou cozidas.

Em sua composição o fruto possui proteínas, carboidratos, fibras, cinzas, $\mathrm{Ca}, \mathrm{Mg}, \mathrm{Fe}, \mathrm{P}$, $\mathrm{Na}$, é fonte de vitamina $\mathrm{C}$ e zinco. Possui ação antioxidante, pois é fonte de compostos fenólicos, além de apresentar inúmeros benefícios ao nosso organismo. Possui ação diurética, dessa forma ajuda a reduzir o inchaço, por possuir carboidratos é fonte de energia, melhora o funcionamento do intestino, ajuda a reduzir as taxas de colesterol, fortalece o sistema imunológico, aumenta a saúde dos ossos, combate o estresse, controla o apetite, ajuda no ganho de massa muscular, previne anemia e melhora a saúde do sistema nervoso.

Outra PANC bem conhecida de algumas regiões é o Jambo-vermelho (Syzygium malaccense (L) Merr. \& L. M. Perry), trata-se de arvore frutífera, onde são consumidos folhas e frutos, podem ser utilizados em sucos, mousses, saladas. Os frutos tem características antioxidantes e as folhas ajudam a controlar a diabetes, pois são fonte de miricitrina, componente antioxidante e anti-hiperglicêmico. Podemos destacar também nesse guia a Jurubeba, outra espécie de PANC onde seus frutos e folhas são consumidos. A jurubeba é bastante comercializada nos estados de GO, MG e MG, sendo vendidos em feiras livres em potes ou em forma de picles (conserva).

Estudos comprovam que a jurubeba possuem alcaloides esteroidais que conferem ação protetora e atua como inibidora do ácido gástrico e tem ação antiulcera. (TESKE; TRENTINI, 1997).

De um modo geral as PANCs em sua maioria apresentam fator nutricional elevado, aumentando para o indivíduo que as consomem a ingestão diária vitaminas e minerais essenciais ao desenvolvimento humano, como visto em alguns casos são capazes de substituir as hortaliças convencionais, podendo ser inserida na alimentação diária, cultivadas em pequenos espaços e jardins, podendo também ser comercializada gerando uma fonte de renda

\section{CONSIDERAÇÕES FINAIS}

O desenvolvimento do presente estudo possibilitou verificar após a leitura realizada que as PANCs são uma opção como complemento a alimentação tanto do ser humano, como de animais, porém, ainda precisam ter estudos relacionados a sua composição mais aprofundados, pois algumas apresentam toxidade que podem trazer prejuízos a saúde quando não utilizadas corretamente. 
Contudo com o pouco que se tem de informação sobre algumas espécies de PANC, podemos confirmar seus benefícios, funcionalidades e sustentabilidade principalmente ligados a ações antioxidantes e anti-inflamatórias, podendo ser consumidas de várias formas, tanto na forma imatura, cozidas ou industrializadas. Além disso, também ser utilizada como fonte de renda, pois muitas dessas PANCs crescem de forma espontânea, e são encontradas com facilidade na natureza.

Ao iniciarmos a busca do material para elaboração desse trabalho, identificamos poucas publicações acerca do tema.

Dessa forma, conclui-se que se faz necessário que os pesquisadores aprofundem seus estudos em relação ao tema, pois existe uma grande diversidade a ser explorado. Por fim, verifica-se a necessidade que a população adquira mais conhecimento para que essas PANCs sejam inseridas na sua alimentação diária.

\section{REFERÊNCIAS}

BRASIL. Ministério da Agricultura, pecuária e Abastecimento. Manual de hortaliças não convencionais. Secretaria de Desenvolvimento Agropecuário e Cooperativismo. Brasília: mapa/ACS, 2010.

COSTA, E.A. Nutrição e Fitoterapia: tratamento alternativo através das plantas. Petrópolis, RJ, Editora Vozes, 2012.

FISCHER, C.G.; GARNETT, T.P. Plates, Pyramids, Planet - Desenvolvimento em Diretrizes alimentares saudavéis e sustentaveis: uma avaliação do estado de jogo. Organização das Nações Unidas para Alimentação e Agricultura, Rede de Pesquisa sobre Clima Alimentar da Universidade de Oxford, 2016.

KELEN, M. E. B.; NOUHUYS, I. S. V.; KEHL, L. C.; BRACK. P.; SILVA, D.B. Plantas alimentícias não convencionais (PANCs): hortaliças espontâneas e nativas. ( $1^{\mathrm{a}}$ ed.). UFRGS,PortoAlegre, 2015.

KINUPP, V. F. Plantas Alimentícias não convencionais (PANC) no Brasil: guia de identificação, aspectos nutricionais e receitas ilustradas. Instituto Plantarum de Estudos da Flora, São Paulo, 2014. 
LIRA, A. Mais do que matos, elas são plantas alimentícias não convencionais (PANCs). Empresa Brasileira de Pesquisa Agropecuária - EMBRAPA. Ministério da Agricultura, Pecuária e Abastecimento. Brasília, 20 abr. 2018. Disponível em: https://www.embrapa.br/busca-de-noticias/-/noticia/33580014/mais-do-que-matos-elas-saoas-plantas-alimenticias-nao-convencionais-pancs Acesso em: mar. 2019.

MARIA FILHO, J. A importância das pancs para promoção da saúde e educação nutricional, social, gastronômica e ambiental. Revista Brasileira de nutrição funcional - ano $15, \mathrm{n}^{\circ} 65$, 2016. Disponível em: https://www.vponline.com.br/portal/noticia/pdf/9d41f4d83c84f6e23d43083c25e7a2b9.pdf Acesso em: 08 de maio de 2019.

PASCHOAL, V.; SOUZA, N.S. Plantas Alimentícias não convencionais (PANC). In: CHAVES, D. F. S. Nutrição Clínica Funcional: compostos bioativos dos alimentos. VP Editora, 2015. Cap. 13. p. 302-323. 2.

PEDROSA, M.W.; MASCARENHAS, M.H.T.; CARVALHO, E.R.O.; SILVA, L.S.; SANTOS, I.C.; CARLOS, L.A. Hortaliças não convencionais: saberes e sabores. Belo Horizonte, 2012. Disponível em: http://www.epamig.br/index.php?option=com_docman\&task=doc_download\&gid=2696. Acesso em: 06 de maio 2019.

TESKE, M.; TRENTINI, A. M. M. Herbarium compêndio de fitoterapia. 3 ed. Curitiba, 1997. 